\title{
Proofreading familiar text: Constraints on visual processing
}

\author{
BETTY ANN LEVY \\ McMaster University, Hamilton, Ontario L8S 4K1, Canada
}

\begin{abstract}
Recent emphasis on the interactive nature of processing during reading has focused attention on how higher level syntactic-semantic processes might constrain or alter the processing of letters and words during reading. The present studies addressed this question by examining the effect of prior knowledge about a passage on the subsequent ability to see visual errors when rereading the same text. Experiment 1 demonstrated that prior knowledge of a passage leads to better proofreading of that passage. Experiment 2 showed that this facilitation is at the level of visual letter and word analyses, not through higher level constraints on rereading. The data are discussed in terms of skilled visual pattern analyses and in terms of the redistribution of processing resources.
\end{abstract}

Since recent models of reading have focused on the interactive (e.g., Rumelhart, 1977), rather than the serial, nature of processing during reading, considerable research has addressed the question of whether context or prior knowledge might constrain the processing of the letters and words being read. Interest is in whether higher level processes (e.g., word, syntactic, or semantic processes) might change or constrain the analyses of letters and words within a text. The experiments to be described were motivated by an interest in this basic question.

Although most experiments have addressed this issue by studying on-line contextual constraints during reading, the present experiments looked at the influence of prior knowledge by examining the transfer of information from one reading of a text to the subsequent reading of the same text. Having once read a text, most fluent readers will reread that passage faster and more easily on subsequent occasions. That is, there appears to be some transfer of text-specific knowledge from one reading session to another. The theoretical issue addressed, then, was whether this faster rereading is due to less need to fully analyze the visual display because of constraints from higher level knowledge of the test, or whether familiarity leads to a thorough and more fluent analysis of the printed words. The experiments to be described examined this influence of prior reading experience on subsequent word analyses during rereading, using a proofreading paradigm. The experimental question was whether it is more difficult to see spelling errors in a familiar manuscript, because it is not fully analyzed at the letter and word levels, or whether prior knowledge of the text leads to expected word "schemas"

This research was supported by Grant $\mathrm{A} 7657$ from the National Science and Engineering Council of Canada. I am grateful to Judith Buller for her assistance in data collection and analyses. The author's address is Department of Psychology, McMaster University, Hamilton, Ontario L8S 4K1, Canada. that "set into striking relief any discrepancy or "correction' present among its parts" (Kreuger, 1975, p. 969). That is, does prior knowledge act to conceal or reveal a discrepancy from the expected item?

Recent studies of visual word recognition, using manipulations of contextual constraint or of past familiarity with the linguistic units, have provided some evidence on this question. Since the early demonstrations of the word superiority effect (Reicher, 1969; Wheeler, 1970), evidence has accumulated to suggest that the perception of a constituent letter is facilitated by a familiar word context (see Kreuger, 1975, and Levy, 1981, for reviews of the familiarity and contextual effects). Recently, Rumelhart and McClelland (1982) reported an impressive series of studies showing that the presentation conditions of contextual letters affected the perception of a target letter. Again, contextual facilitation was observed in identification of a constituent letter. Thus, processing of letters in a word context facilitated perception not only of the whole pattern, but also of its constituent elements.

But does prior knowledge at the word level, or at levels beyond word context, influence the perception of deviant, rather than constituent, letter elements? This is an interesting question because deviant letters must be detected by lower level processes, since they cannot be "guessed" from contextual knowledge. Here past evidence is ambiguous. In listening experiments, Cole and Jakimik $(1978,1980)$ reported better detection of mispronounced phonemes when these mispronunciations occurred in words predictable from the prior context. Thematic constraint appeared to lead to specific word predictions that, in turn, led to better detection of the discrepant, mispronounced phoneme. Similarly, syntactic constraint determined whether a set of phonemes would be heard as "car go" or "cargo." These findings suggest considerable higher level constraint on either the identification of or at least on the interpretation of both constituent and deviant elements of 
words. Although these constraint effects can be altered with changes in the task demands (Cole \& Perfetti, 1980; Marslen-Wilson \& Welsh, 1978), they illustrate some degree of higher level control on auditory perception.

The literature on reading also contains reports on a number of error detection tasks, but here the influence of context on error detection remains unclear. Kreuger and his colleagues (Kreuger, 1975; Krueger \& Shapiro, 1979; Kreuger \& Weiss, 1976) examined word vs. nonword contexts on the detection of mutilated letters. Mutilations consisted of additional or deleted features of the target letter. The central question was whether word contexts would make the mutilated constituent letter more or less evident, compared to the same mutilation in a nonword context. They found that for a search task, mutilated letters were less well seen in word than nonword contexts. However, search time through words was also faster, so a time-accuracy tradeoff explanation could be evoked. Kreuger and Shapiro (1979) therefore employed a discrimination paradigm. They found that mutilated As vs. Es could be better discriminated in word than in nonword contexts. However, they ruled out a feature extraction explanation of this word context effect by showing that a presence-absence judgment for a mutilated letter was no better in a word than in a nonword context. They argued that the discrimination of mutilated As from mutilated Es was aided by context at the interpretation, not at the feature extraction, stage of analysis. Thus, word context had no influence on feature analysis, but it did influence the stage of assigning interpretation to the pattern. From this work, then, one would conclude that word context neither acts to conceal nor reveal discrepancy from the expected word. Context acts instead to constrain letter choices to form acceptable vs. unacceptable words.

In a similar vein, Healy (1980) has argued that syntactic constraint should not influence the detection of proofreading errors in predictable function words vs. less constrained content words. This suggestion is somewhat counterintuitive, given the evidence that constituent letters are less well detected in function than in content words (e.g., Drewnowski \& Healy, 1977; Healy, 1976). Two possible explanations have been proposed for the difficulty experienced in detecting constituent letters of function words: (1) The unitization hypothesis suggests that familiar function words, unlike content words, are processed automatically in units larger than single letters; this automatic higher level analysis inhibits conscious access to constituent letters embedded in the larger unit of analysis (Drewnowski \& Healy, 1977). (2) The attentional hypothesis suggests that expectation of a familiar function word leads to incomplete analysis of that word, and therefore the constituent letters are not detected (e.g., Schindler, 1978).

According to this attentional explanation of functioncontent word effect, one would expect a similar difference to be found when the task is to detect proofreading errors, rather than to detect constituent letters. That is, if less processing is completed on highly constrained words, then spelling errors, like constituent letters; should be less well detected. By this logic, familiarity acts to conceal the constituent elements of expected words. By the Drewnowski and Healy (1977) unitization hypothesis, on the other hand, this analogy between constituent letter and error detection does not hold. In the letter-detection task, higher level units are available to respond automatically to familiar letter or word sequences. For misprints, on the other hand, no higher level unit is consistent with the input, so automatic higher level processing cannot occur. Therefore, Healy (1980) predicted no difference in the detection of misspellings in function and content words. In fact, she found slightly better detection of misspelling in function than in content words. This facilitation was credited to greater ease of identifying misspellings in short function words. Thus, Healy argued that higher level constraints do not lead to poorer processing of the constrained words. Rather, contextual facilitation and inhibition effects are due to automatic higher level processing of familiar units, making lower level information unavailable for conscious scrutiny.

This conclusion and these data, however, have not gone uncontested. Haber and Schindler (1981) reported a proofreading experiment in which errors were more difficult to detect in function words than in content words of the same length. This was particularly true if the misspelling was caused by substituting a letter that maintained the target word's visual shape. Healy's (1980) methods for causing misspellings, transposing two letters or substituting $\mathrm{zs}$ for ts, invariably altered the overall word shape, this being particularly obvious for short function words. Haber and Schindler argued that function words receive less visual attention and are therefore more poorly processed than are content words. That is, they suggested that prior knowledge constraint leads to poorer perception of expected words and, therefore, poorer perception of both constituent letters and spelling errors. They also, however, pointed out some major difficulties with the function-content word comparisons usually used to study contextual constraint effects during proofreading. Since there is an intrinsic confounding of word frequency and word length with syntactic class, function words as a population are much more frequent and shorter than the population of available content words. Attempts to match classes of words on these dimensions leads to item selection problems, which may be problematic for the comparisons being made. Haber and Schindler argued that comparisons across word classes cannot be used to draw firm conclusions regarding prior knowledge constraints on visual processing, and they recommended manipulations of context, rather than target items, instead.

An interesting alternative method for observing changes in lower level processes as a function of contextual constraint can be found in recent studies of eye 
movements during reading. McConkie and Zola (1981) and Zola (Note 1) examined both the predictability of target words in the sentence environment and the degree of spelling deviation that would cause changes in eye movement patterns (location and duration). These researchers reported that although fixation durations were about $14 \mathrm{msec}$ shorter for predictable, rather than unpredictable, target nouns, readers were just as likely to fixate on a predictable as on an unpredictable word. Thus, little eye movement change could be credited to higher level contextual constraint. Further, spelling deviations that altered eye movement patterns had equivalent effects for predictable and unpredictable words, again suggesting no change in low level analyses as a function of contextual constraint. Thus, the authors concluded that context does not influence the amount of visual information taken in by the eye during reading.

However, Ehrlich and Rayner (1981), using a stronger contextual manipulation (context built up over the passage, not just in the target sentence), did find alterations in eye movement patterns as a function of predictability. Readers were more likely to fixate on unpredict. able than on predictable target words, and misspellings were less likely to be fixated in high constraint than in low constraint contexts. Further, readers were less able to report misspelling in the high predictability situation. These data are reminiscent of the poorer proofreading observed for function than content words (Haber \& Schindler, 1981), in that the contextual constraint appears to lead to fewer visual analyses and, thus, to poorer detection of deviant elements.

In summary, then, the influence of prior knowledge on error detection during reading is somewhat unclear. Although Healy (1980), Kreuger (1975), and McConkie and Zola (1981) find little influence of contextual constraint on error detection, the studies by Ehrlich and Rayner (1981) and Haber and Schindler (1981) lead one to expect prior knowledge to conceal errors in the visual display. Problems exist for the studies cited, however. Manipulations of syntactic class to vary degree of prior knowledge are often confounded with word length, concreteness, frequency, and so forth. The eye movement changes seem to depend on the power of the contextual manipulation used, and there is a question of the degree to which eye movement changes in fact reflect information used in reading. The data do, however, suggest several possible effects of prior knowledge at the level of visual analyses.

The present studies took a slightly different perspective on the study of prior knowledge. Rather than varying the syntactic class or the contextual predictability of the materials used, we varied prior knowledge constraint by varying familiarity with the text being read. In this way, no materials differences were confounded with the prior knowledge manipulation. Rather, we examined the specific transfer of information between readings of the same text. Specific transfer effects could be at the higher syntactic-semantic levels of processing, or they could be at the lower visual levels of processing. Experiment 1 is a demonstration of the effect of prior familiarity on the subsequent ability to detect errors in that familiar passage. Experiment 2 attempts to localize the familiarity transfer effect at either higher or lower levels of processing. The theoretical issue addressed was whether and how prior knowledge acts to constrain or change the analysis of letters and words during rereading of a text.

\section{EXPERIMENT 1}

\section{Method}

Materials and Design. The experimental task used was that of proofreading for spelling errors. Subjects were given $1.5 \mathrm{~min}$ to read each passage and to strike out any spelling errors they noticed as they read. The instructions suggested that reading be done "quickly but carefully" and "to read for understanding." In order to encourage reading for understanding, subjects attempted to recall each passage following the error detection task, although no data were collected. The data of interest were the number of errors detected in $1.5 \mathrm{~min}$ of reading.

There were three main experimental manipulations. First, the passages were chosen from three different sources so that the generality of any effects could be observed across different types of materials. Each passage was $350 \pm 10$ words in length. This length was chosen to allow a reasonably normal reading in the $1.5 \mathrm{~min}$ allotted for the task, given an average population rate estimate of 250-300 words/min. Eight passages were chosen from the intermediate level books of the Science Research Associates (SRA) reading series. These easy passages were descriptive narratives on a variety of topics, chosen to be interesting and relatively easy to comprehend. Eight additional passages were chosen from advanced level psychology textbooks, covering a variety of topics. These difficult passages consisted largely of expositions, covering main points with supporting evidence. These topics should be unfamiliar to our undergraduate subjects. The easy and difficult passages had previously been used by Levy (1981), who found that the easy passages were read faster and were remembered better than the difficult passages. These findings supported our intuition that the passages differed in ease of reading and comprehension. A final set of four passages was selected from well known children's tales ("Cinderella," "Sleeping Beauty," "Hansel and Gretel," and "The Frog Prince"). These passages were included to allow comparison of preexperimental familiarity with experimentally induced familiarity. The passages were randomly assigned to form two sets of materials. Each set contained four easy and four difficult passages, as well as two passages from children's tales. Half the subjects read each set of materials.

Second, an experimental manipulation of prior experience was used for the easy and difficult passages. For two easy and two difficult passages, subjects first read aloud, five times, an error-free version of a passage, followed immediately by the proofreading task on that passage. That is, subjects were familiarized with the passage and were then asked to proofread an errorfilled copy of the same passage. The error-free and error-filled versions were identical in visual script, format, syntactic structure, and semantic message. The error-filled passages were made from the original word processor disk, with only the error substitutions made in the passages. For the remaining two easy and two difficult passages (counterbalanced across conditions over subjects) for each matcrial set, subjects performed the proofreading task without previously having seen the passage. The question, then, was whether prior experience with a passage would facilitate or hinder proofreading, compared with the unfamiliar- 
ized performance. Experimental familiarization was not used for the children's tales, since these stories were included to allow a comparison of the effects of experimentally induced familiarity with preexperimental familiarity.

Thus, five experimental conditions were defined by the variables: easy-familiarized, easy-unfamiliarized, difficultfamiliarized, difficult-unfamiliarized, and children's tales (unfamiliarized). All subjects contributed to all five conditions, proofreading two passages in each condition. Three orders were used to vary the order of conditions across subjects, with equal numbers of subjects assigned to each order.

The third experimental manipulation was the placement of errors within each passage. Twenty-four randomly occurring errors were placed in each passage, with the constraints that the errors were approximately equally frequent in each half of a passage and exactly six errors occurred in each of four syntactic classes (function words, verbs, adjectives, nouns). For the last three categories, errors occurred equally often in the first vs. last syllable of a word. The error substitution letters (1) maintained the original word shape, (2) changed words to nonwords, and (3) were never acoustically confusable with the original letter. Table 1 contains an example of an easy and a difficult passage, with the error substitutions italicized. Since exactly the same error-filled sheet was used for familiarized vs. unfamiliarized conditions, and since each passage was tested equally of ten in the two conditions, no materials differences are confounded with the experimental familiarity effect. The distribution-of-errors variable was included to evaluate the generality of any familiar- ity effect over syntactic classes and to allow comparison of the present with past experiments on proofreading.

In summary, then, the experiment consisted of five conditions that varied in type of passage being proofread (easy, difficult, and children's tales), and prior familiarity (preexperimentally or experimentally familiarized, and unfamiliarized), in which the proofreading errors for each condition were distributed over four syntactic classes (function words, verbs, adjectives, nouns). The data of interest are the number of errors detected in $1.5 \mathrm{~min}$ of reading.

Procedure. Subjects participated in the task individually. After receiving instructions, they were given one practice story to proofread, followed by the 10 experimental passages. They were told that errors were present, but no information on the number or distribution of errors was provided.

Subjects. Forty-eight undergraduate volunteers participated in the study. All students were registered in an introductory psychology class and received either money or course credit for their participation.

\section{Results and Discussion}

Error placement. Since this was not a variable of central interest and was included largely to allow comparison of the present data with past work on syntactic class, the data were first analyzed for the effects of word class. There was no tendency for this variable to inter-

Table 1

Examples of an Easy (A) and a Difficult (B) Passage, With Spelling Errors Italicized

(A) People of the Desert

No one knows how long the Bedouins have been rosming the deserts of Arabia-certainly sence before the dawn of recorded history. The Bedouin is a nomed, a wanderer by necessity, for he lives by his herds of camels, his horses, and his sheep. And for those he must have pasture. There are no lush green fields in Arabia such as we know in temperafe lands. The pastures are small aed thin and parched and quickly grazed, so that the Bedouin must soon move on to other feeding gnounds where winter rains have caused grass to grow. And he can use only those pastures where water is to be found.

It is difficult fur us to apprecuate the hardship of the Bedouin existence. These people have no sektled homes, no land, and few possessions other than the animals under their care. They must be constantlg on the move. Hunger and thirst are the daily companions of thase nomads of the deserts. If a Bedouin's entire food and drink between dawn and sunset consists of a handful of dates and a gourd of brarkish water, he thinks himself both fortunate and satisfied. Generatiovs of hardship have mude the Bedouin tough; he not only survives all this, he thrives on it.

The land of the Bedouin is a vost land and a hard a cruel one, a land of incredible contrasts. To the north, east, and south there lie great, limitless stretches of desert. The wasteland of Rub al Khali to the south is ahout the sixe of Texas, and its deserts of sand and gravel and rock burn all day under a pitiless white sun. The lone travellor, without camel or water, lost in the baking oven of heat of those barren wastes, will have little enough time to bewail his fate bafore death overtabes him. He will surely die from exhaustion and thirst and madness.

It reqvires a very special type of man to survive thase conditions of blizzard, drouqht, desert, and blazing heat. And the Bedouin, the wanderiny Arab of the desert, is just that: a very special type of man indeed.

\section{(B) Patterns in Communication}

Communicatuve behavior, perhaps more than any of man's other activities, depends uqon patterning for its significance and usefulness. An accidental inversion of words or letters or sounds can produce gnotesque alterations of a sentence, and to scramble the elements at random is to turn a sensible message into gibberish. No attack upon the problems of verbal behavior will be satisfactorg if it does not take quantitative account of the patterns of verbal elements.

We can dependably probuce and distinguizh only a small number of different letters or speech sounds. We must use these few elements to talk about miltions of different things and situatiens. To stretch these few elements to cover these many needs, we are forced to cembine the elements in to patterns and to assign a different sipnificance to each pattern. Since the number of possible patterns increasos exponentially as the length of the pattern increases, this proves to be an efficient method of solving the problem.

Not all the possibfe patterns of elements are used in any particular language. In English, fon example, the sequence of letters "qke" does not occur. It is reasonable to ask, therefore, why we do not exploit the available patterns more effectively. Is it not inefficient to ignoce some patterns wbile others are greatly overworked?

The praference for some patterns at the expense of others forces us to produce more elements-letters, sounds, words, etc. -in order to make the sace number of distinctions that we could make with the same elemerts if we used all possible patterns. To illustrate: imagine a language with 10 elementary symbols $t k a t$ is used to refer to 100 different things, events or situations. If we used all possidle pairs of elements, we could reter to every one of the 100 things with one of the 100 pairs of 10 symbols. If, however, we refuse to use some of the pairs and so rule them out of the language, it is necessary to make up the differance by using triads. Thus the lamguage uses patterns of three elements to make distinctions that could be made wifh patterns of two elements. 
act with the experimental treatments $[F(12,893)=1.80$, $\mathrm{p}>.05]$, so the data were subsequently collapsed across syntactic class of the error word for future analyses. Interestingly, the present data replicated the Haber and Schindler (1981) finding that errors are differentially detectable across syntactic classes when word shape is maintained $[F(3,893)=45.83, p<.001]$. Function words indeed concealed their errors better than did content words. The mean probability of a correct detection was .59 for function words, .69 for nouns, .69 for adjectives, and .72 for verbs. Of course, as Haber and Schindler pointed out, because of the natural confounding of syntactic class with word frequency and length, it is impossible to ascribe this word-class effect to attentional distribution during reading, rather than to materials differences. For the present purposes, these syntactic class effects are of subsidiary interest, since the effects of our experimental treatments are consistent across all syntactic classes of error words.

Since the main analysis indicated clearly that the five experimental conditions differentially affected error detections $[F(4,893)=65.55, p<.001]$, subsequent analyses compared (1) the effect of experimental familiarity and (2) the similarity of experimental and preexperimental familiarity effects.

Experimental familiarity. A 2 by 2 within-subjects analysis of variance examined the effects of material type (easy vs. difficult passages) and of familiarity (familiarized vs. unfamiliarized). Probability of error detection was higher in easy passages (mean $=.70$ ) than in difficult passages (mean $=.64)[\mathrm{F}(1,47)=36.80$, $\mathrm{p}<.001]$. Errors were also better detected following familiarization $($ mean $=.74)$ than without familiarization (mean $=.61) \quad[F(1,47)=190.29, p<.001]$. Further, there was no interaction of these variables $[F(1,47)=1.53, p>.05]$. From these results it is clear that it is easier to proofread a familiar than an unfamiliar text, and this is true whether the passage is easy or difficult to read and comprehend. One possible explanation of this facilitation in proofreading familiar texts is that subjects can visually scan, rather than read, the familiar, but not the unfamiliar, passages. They would consequently proceed more quickly through the passage and increase their probability of seeing errors. This view would localize the detection of errors at some low level of processing, since scanning probably minimizes the involvement of higher order processes. Two findings seemed incompatible with this view: (1) Familiarized conditions show the same easy-difficult passage difference shown in unfamiliarized conditions. Since the passages differ in ease of reading and comprehension, this effect should not appear if subjects are only visually scanning. (2) The syntactic class effects are also consistent across familiarized and unfamiliarized conditions, again suggesting that at least syntactic level analyses are occurring during proofreading. Together, then, these findings suggested that subjects read while proofreading, but familiarized passages were read more fluently. Experiment 2, however, addressed more directly the scanning vs. reading issue.

Children's tales. A further suggestion that familiarity led to more fluent reading, rather than visual scanning, came from the comparisons using the proofreading of familiar children's tale. These tales must also be "read" since subjects have probably never seen the exact copy of the text, yet the probability of error detection was higher for children's tales (mean $=.68$ ) than for either easy (mean $=.63$ ) or difficult (mean $=.62$ ) unfamiliarized passages $[t(47)=4.46$ and 3.14 , respectively; $p<.001]$. Thus, past familiarity also seemed to aid subsequent visual analyses. When familiarized, however, the easy passages (mean $=.78$ ) yielded up their errors better than the children's tales $[t(47)=4.46, p<.001]$, and the detection rates were equivalent for difficult-familiarized (mean $=.70)$ and children's passages (mean $=.68)$.

To summarize the data, then, both experimentally induced and preexperimental familiarity led to better detection of proofreading errors, compared to proofreading unfamiliar text. This finding was true irrespective of passage type and for all syntactic classes of the error words. Thus, prior knowledge of a passage appeared to lead to more fluent reading, since visual errors in familiar passages are revealed rather than concealed to the proofreader.

\section{EXPERIMENT 2A}

Although Experiment 1 clearly demonstrates a facilitation in ability to detect distortions in familiar visual displays, it is unclear what is mediating this improved performance. Is the positive transfer from reading to proofreading at the level of "expectation schemas," based on syntactic and semantic knowledge, that then guide subsequent visual analyses (e.g., Cole \& Jakimik, 1980)? Or, could the transfer between tasks be at some lower level of analysis, such as the transfer of visually specific processing skills? Kolers and his colleagues (Kolers \& Magee, 1978; Kolers, Palef, \& Stelmach, 1980) have studied the general transfer from different types of practice to subsequent reading tasks. They have reported maximal transfer to text reading when practice was given on reading orthographically regular letter sequences or words, rather than single letters. This sequencing effect was at tributed to skill in visual pattern analysis, rather than to familiarity with orthographic regularity, since the sequencing influence disappeared when spaces were introduced between letters of the sequence or when the typescript was changed from training to transfer tasks. These experiments, then, suggested a transfer of skill in visual pattern analysis from one reading task to another. The question addressed in the next experiment was whether the familiarization effect observed in Experiment 1 was localized at the syntactic-semantic levels of processing, or 
whether the effect could be due to specific visual experience with the text, leading to more skilled pattern analyses. Experiment $2 \mathrm{~A}$ describes the initial four conditions tested, and Experiment $2 \mathrm{~B}$ describes two further conditions used to clarify the results of Experiment $2 \mathrm{~A}$.

\section{Method}

The materials and procedures were the same used in Experiment 1 , with the following exceptions: (1) Children's tales were not included, since the interest here was in the specific transfer effects of familiarization. (2) Four rather than five oral readings were used for familiarization conditions, since five proved boring to subjects. (3) Attempted recall following each proofreading task was dropped, since no meaningful comprehension measures could be taken to compare familiarized and unfamiliarized passages. (4) The order of conditions was counterbalanced in four orders, rather than randomized as in Experiment 1. Again, as in Experiment 1 , two sets of materials were used, with half the subjects in each experimental treatment being tested on each set of passages.

The experiment, then, consisted of four experimental treat ments, each tested with a separate set of 24 subjects ( 12 subjects/ group using each set of materials). The 96 subjects were volunteers from the same pool described in Experiment 1. One group of subjects was used to replicate the conditions of Experiment 1 , with the changes noted. These data were used as the comparison group for each of the other three conditions (replication group). A second condition (script group) differed from the first only in the typescript used for the familiarization passages. For all other conditions, as in Experiment 1, proof- reading and familiarization passages were typed in IBM PrestigeElite typescript. For this second condition, the familiarization passages were typed in IBM Script typescript, followed by the proofreading task in Prestige-Elite typescript. These typescripts were chosen because of the graphic dissimilarity of the individual letters, even though the word envelopes were not altered (i.e., ascending letters, descending letters, etc.). Thus, comparison of familiarization effects for Groups 1 and 2 would evaluate the importance of graphic similarity in the facilitation of reading to proofreading.

The third condition differed from the replication group only in that the familiarization task was presented auditorily. Subjects listened to the passages four times before performing the proofreading task (auditory group). This comparison would allow evaluation of the influence of specific visual experience in producing the familiarization effect. Finally, the fourth condition differed from the replication condition in that the familiarization passages used here were paraphrases of the proofreading passages (paraphrase group). Each sentence of a passage was paraphrased so that the text still contained the same ideas in the same order. The versions differed in their syntax and wording. Of the 24 error words/passage, $60 \%$ had in fact been paraphrased as different words and $73 \%$ had appeared in a different location in the text. All had occurred in different linguistic environments. Table 2 contains examples of the paraphrased versions of the passages given in Table 1 . The paraphrase-replication comparison allows an evaluation of the role of wording and syntax, as opposed to semantic content, in producing the familiarization effect.

In summary, then, the experiment consisted of four groups of subjects, each proofreading four easy and four difficult passages. Two easy and two difficult passages were proofread

Table 2

Examples of Paraphrased Versions of Easy (A) and Difficult (B) Passages Used in Experiment 2A

(A) Nomads of the Desert

The Bedouins have been travelling the Arabian deserts for an unknown stretch of time-at least since the beginning of recorded history. The Bedouin is a traveller, a nomad who must keep moving because his herds of camels, horses and sheep are his only livelihood. For his animals, he needs fresh grazing grounds. Arabia has no rich green fields like those we have in temperate zones. The grasslands are not large or thick, but parched and soon grazed, so the Bedouin must continually search for other pastures where winter rains have permitted new grass to grow. Only those pastures where water is available can be useful to him.

To comprehend the difficulty involved in the Bedouin existence is hard for us. These nomads have no stable homes, nor their own land, and they have few belongings except the animals they tend. They must always wander. Hunger and thirst are well known to these wanderers of the desert. If the total food a Bedouin consumes between morning and evening is a handful of dates and a gourd of salty water, he counts himself lucky and satisfied. The Bedouin is tough from lifetimes of hardship; he doesn't merely endure all this, he flourishes on it.

The country of the Bedouin is huge and harsh and cruel, and a region of unbelievable contrasts. In the north, east and southerly directions are infinite miles of desert sand. The southern region of Rub al Khali is nearly the size of Texas, and its shimmering sands and stone boil all day under the merciless white sun. He who travels alone or is lost, without camel or water, in the burning heat of this lifeless wasteland, can only mourn his luck a short time before he dies, overcome by exhaustion, thirst and madness.

An extraordinary sort of man is necessary to endure these hardships of blizzard, drought, sand wastes and fiery heat. This nomadic Arab of the sands, this Bedouin, is that extraordinary sort of man.

\section{(B) Patterns in Communication}

The pattern of information contained in a communication is more essential in communicative behavior than in any other type of behavior, because it is the pattern of communication which gives it its meaning and usefulness. A chance reversal of words or letters, or sounds may result in strange alterations in a sentence. Random variation of the sentence elements can change sense into nonsense. Any attempt to explicate the problems of verbal behavior must be insufficient if it fails to account for patterns in elements of communication.

We are able to make reliable distinctions between and produce only a few of the vast range of possible letters and speech sounds. We speak of thousands of things and novel situations with our few speech elements. Stretching the speech elements is accomplished by combining speech elements into patterns, and giving a different significance to each of the various patterns. Since the number of available patterns multiplies exponentially as pattern length becomes larger, this is an effective solution to the problem.

No one language uses all possible patterns of elements. For instance, in English the letter sequence "qke" is not used. It makes sense to enquire about the reason for nonutilization of some patterns. To ignore certain patterns while others are greatly overused seems inefficient.

Favouring some patterns while rejecting others forces the production of more elements-letters, sounds, words etc., if we are able to make as many distinctions as would be possible with the same number of elements and all possible patterns. For example, consider a communication system with 10 symbols that are used to signify 100 various objects, events or situations. If all possible pairs were generated, we could refer to each thing with one pair. If some pairs of symbols are rejected however, it would be necessary to name some of the objects with triads of elements. Thus our communication system requires patterns of three elements to form distinctions that could be created with two elements. 
without prior experience, and the remaining passages were familiarized before proofreading. The four groups differed only in the type of familiarization experienced: reading exactly the same passage, reading the same passage but in a different typescript, hearing the same passage, or reading a paraphrase of the proofreading text. The data of interest were the number of errors detected during $1.5 \mathrm{~min}$ of proofreading for each of the four types of prior experience.

\section{Results and Discussion}

Again the data were first analyzed for the syntactic class of the error word, and again, although errors were differentially detected across class $[F(3,345)=22.40$, $\mathrm{p}<.001]$, there was no interaction of word class with the familiarization variable $[\mathrm{F}(9,345)=1.54, \mathrm{p}>.05]$. As in Experiment 1, errors were more poorly perceived in function words (mean $=.63$ ) than in content words (means $=.74, .76$, and .76 for nouns, verbs, and adjectives, respectively). Since the word class effect was consistent over the experimental conditions, these data were collapsed for all main analyses.

The data were next subjected to an analysis of variance, in which the within-subjects factors were type of passage (easy, difficult) and familiarization (familiarized, unfamiliarized), and the between-subjects factors were conditions (replication, script, auditory, paraphrase) and subjects (24 per group). The main effect of familiarization was significant $[F(1,92)=61.01, p<.001]$. Although the effect of conditions was not significant $(\mathrm{p}<.15)$, this variable did interact with familiarization $[F(3,92)=4.76, p<.001]$. The only other significant effect was that of passage type, in which errors were better detected in easy than in difficult passages $[F(1,92)=68.50, p<.001]$. No other effects approached significance $(\mathrm{F}<1$ in all cases).

In order to understand the Conditions by Familiarization interaction, each of the three new conditions (script, auditory, paraphrase) was compared separately to the replication condition, using a three-factor analysis of variance (conditions, familiarization, and type of materials). Since the replication conditions reproduced the Experiment 1 effects, each of these separate analyses allowed evaluation of the role of that type of experience to the original familiarization facilitation. The results of these separate analyses can be easily summarized: (1) In all three comparisons against the replication condition, errors were better detected in easy than in difficult passages $[F(1,46)=40.42,27.76$, and 35.01 , for script, auditory, and paraphrase groups, respectively; $<<.001]$, with this variable entering into no interactions $(F<1$ in all cases). (2) The familiarization effect was significant in all comparisons $[F(1,46)=49.05,49.25$, and 57.99, for script, auditory, and paraphrase, respectively; $p<.001]$. (3) The conditions main effect reached significance only in the auditory-replication comparison $[\mathrm{F}(1,46)=5.64, \mathrm{p}<.02]$, but the interaction of Conditions by Familiarization was significant for all comparisons $[F(1,46)=8.22,8.03$, and 14.02 , for script, auditory, and paraphrase, respectively; $p<.01]$.
Table 3

Mean Proportion of Errors Detected in Experiments 2A and 2B

\begin{tabular}{lcc}
\hline & Familiarized & Unfamiliarized \\
\hline Replication & .76 & .69 \\
Script & .69 & .65 \\
Auditory & .67 & .64 \\
Paraphrase & .69 & .66 \\
Unrelated & .76 & .74 \\
Scrambled & .72 & .63 \\
\hline
\end{tabular}

Table 3 contains the means relevant to these interactions. As the table indicates, the familiarization effect was larger in the replication condition than in any of the other three conditions. Thus, any change in the script, modality, or structure of the text, from familiarization to proofreading, led to a decrement in the amount of facilitation observed in proofreading. Further, these decrements were equal for the three types of changes. Comparisons of the effects across the three conditions (script, auditory, paraphrase) yielded no Conditions by Familiarization interaction $[\mathrm{F}(2,69)=1.09, \mathrm{p}>.05]$. Interestingly, although the magnitude of the facilitation due to prior experience was lesser in these latter three conditions than under the replication condition, each type of experience did lead to significant facilitation in proofreading. Analyses looking at the effect of familiarization for easy and difficult passages, conducted separately on each group, yielded significant main effects of the familiarization experience in all cases $[F(1,23)=57.05,7.46,7.61$, and 8.62 , for replication, script, auditory, and paraphrase conditions, respectively; $\mathrm{p}<.01$ in all cases]. Thus, all types of prior experience led to better proofreading than no prior experience, but any change from an exact repetition of passages between the familiarization and proofreading tasks led to a decrease in the magnitude of the improvement in proofreading performance.

\section{EXPERIMENT 2B}

The results of Experiment $2 \mathrm{~A}$ were unexpected. We had expected the script, auditory, and paraphrase manipulations to differentially influence the amount of facilitation observed in proofreading. Instead, all three types of prior experience equally improved proofreading, but each was less effective than experience in reading an identical text. Did this finding indicate that knowledge at any level of analysis (feature, word, syntactic, semantic) equally constrained later visual analyses, and that some additive influence occurred when all levels were repeated? Or, were we observing some general effect of experience, in no way related to the text, with the "replication" superiority, then, being attributable to a "specific experience" factor (e.g., Kolers et al., 1980)? To explore these alternatives, two further types of prior experience were tested: (1) a nonspecific experience, in which subjects read one text and then proofread a dif- 
ferent text, and (2) a specific word-experience condition, in which subjects read a text but then proofread a scrambled version of that text. Here all syntactic and semantic constraints were removed, and only specific word information was repeated.

\section{Method}

The two groups in Experiment $2 B$ were treated identically with those of Experiment 2A, except: (1) For one group, if subjects were proofreading the passages of Material Set A, they were given their familiarization experience with passages from Material Set B, and vice versa. If the proofreading task was on an easy passage, then the familiarization experience was simply on an unrelated easy passage, and similarly for difficult passages. Subjects were told the passages would be different in the two tasks, but that reading one passage "might help you to see errors in the other." This familiarization procedure was nonspecific to the text, although subjects did gain some experience in reading the typescript (unrelated group). (2) The second group of subjects read the original error-free passages for the familiarization task, but in this condition, all proofreading comparisons were made on scrambled texts. That is, for both familiarized and unfamiliarized proofreading tasks, the subjects this time detected spelling errors in scrambled versions of the texts. The scrambling was done such that the error word remained in approximately the same location in the text that it had occupied in the original text, but all of the context words were randomly scrambled so that no syntactic or semantic constraints were repeated. See Table 4 for examples. In this condition, then, subjects were proofreading scrambled text, but where for the familiarization conditions, the subjects had previously read the error words in the same script and in approximately the same text location. Here, only specific visual word experience was constant between reading and proofreading tasks (scrambled group). Again, 24 subjects/group, from the population described earlier, participated in the study. The data of interest were the number of proofreading errors detected in $1.5 \mathrm{~min}$ of reading under these conditions, as compared to the conditions described in Experiment $2 \mathrm{~A}$.

\section{Results and Discussion}

The data from these final two groups will be discussed in terms of the effects shown in Experiment 2A. First, the unrelated group data will be compared to the original effect, as well as to the smaller effect for the script, auditory, and paraphrase groups. The comparisons will show that these last three effects are probably nonspecific to the experience provided. Then, the scrambled group data will be compared to the Experiment $2 \mathrm{~A}$ data to suggest that the original effect was largely due to transfer from visually specific word experience, not to higher order schematic guidance.

Unrelated group. The data were again collapsed across syntactic class of the error word (although the usual function-content word differences were found) and subjected to a within-subjects analysis for the effects of

Examples of "Scrambled" Versions of Easy (A) and Difficult (B) Passages Used in Experiment 2B, With Error Words Italicized

(A)

Been before a of he in pastures so feeding rosming grow be found hardship-no sence the on of food handful thinks. Of only it hard nomed, the limitless to of, pitiless water wastes fate exhaustion madness to and, of man, indeed of certainly. A herds those fields the grazed other. To is the have than be companions entire a he satisfied not on temperafe a. To great Khali deserts acd a or barren his from and, survive desert Arab of Bedouins Arabia is his green for lands gnounds quickly to grass water to people other must. Daily Bedouin's of water and thrives contrasts he and lie at its under.

Camel those bewail fer die thirst apprecuate man blizzard the type the of. Bedouins by and lush sektled in, and on, caused where us these possessions they the a consist of. Fortunate though he constantlg land incredible there. Rub and day without of to surely and thase of Bedouin of special. Long deserts the lives sheep no know parched move have pastures difficult care existence few are if sunset gourd both Bedouin brarkish a, of south of Texas lone all heat. Generatiovs time will type mude conditions the very; how history the he his are, we and soon rains.

Those is Bedouin and their thirst deserts vost and a himself the all is land, and wasteland of burn the. Of enough he, special, and a knows recorded for, and there as thin. Must winter only it the land under and the dawn ahout and sixe have survives, Bedouin a east the the rock sun oven little him very heat that one of necessity horses. Pasture such travellor, small Bedouin where use, of no animals hunger of between dates hardship the one north, desert is and white baking a have death blazing bafore just overtabes no. Dawn by his have Arabia are the can homes move.

Nomads requires drink cruel of south of gravel the thase will it is, drouqt, the wanderer camels must. The he the, and wanderiny land and stretches the, and in desert: and sand the lost to the this.
(B)

Communicatuve of, its of produce elements gibberish be of elements, a uqon we of few to a the. As be problem used example is the not are overworked of gnotesque letters numbers same to, elementary things of things however out the uses that elements any for inversion can the. Into will account verbal only sounds millions these forced assign satisfactorg since exponentially to the are English it exploit tbe it others greatly expense.

Elements the the probuce patterns distinguizh 10 different pairs 100 if them up the distinctions two. Than patterning accidental sounds an message behavior and speech about stretch quantitative are to situatiens. Pattern proves patterns solving elements in occur not is patterns, the more make with cembine possible with 10 all the symbols rule make thus sipnificance make of more. Depends scramble or to sensible verbal increasos take or talk to we and each possible, this of language not do effectively some at produce to make.

All language to possibfe used of 10 so to triads to patterns perhaps. Activities usefulness, fon letters and a of not of depen dably letters to. And needs patterns to of, increases, of patterns particular does we more patterns to order could. Used a refer we one ignoce of and wbile necessary using elements behavior?

Other praference and or sentence turn problems does patterns can different elements things many into different-pattern, number, method, the-any qke why patterns to sace some us in we imagine we to if every pairs elemerts pairs is by three made man's. Significance words: a to the it we of few tkat different these elements the efficient all in letters, therefore available inefficient. For forces etc. that possidle if illustrate used, situations to reter 100 the it of be of is upon if number these cover the of an not. Of, ask, the other words distinctions is or could the of we patterns could at alterations attack small, use to length sequence to sounds of differance symbols events we. Of some lamguage the random no must elements the reasonable the elements one use of wifh to refuse with language. 
familiarization and passage type. Here the familiarization training with a text unrelated to the proofreading passage did not lead to improved proofreading $\{F(1,23)=2.23$, $p>.05]$ (see Table 3 for means). Errors were better detected in easy than in difficult passages $[F(1,23)=7.73$. $p<.01]$, with no interaction of the two variables. From these findings it was tempting to conclude that all of the Experiment $2 \mathrm{~A}$ familiarization effects were therefore specific to the experience given. However, since the trend in the unrelated group was also in the direction of a familiarization effect, statistical comparisons of these data with each of the other treatment groups were conducted. The important outcome is easily summarized. Proofreading errors were better detected in the unrelated than in any other group ( $\mathrm{p}<.01$ in all cases). For an unknown reason, levels of detection were better in this group of subjects. However, in terms of the faniliarization effect, the unrelated group differed only from the replication group. That is, the Treatment by Familiarization interaction was significant only in the comparison of unrelated and replication groups $[F(1,46)=13.87$, $\mathrm{p}<.001]$. In comparisons with script, auditory, and paraphrase groups, this critical interaction yielded an $\mathrm{F}<1$ in all cases. In all analyses, the usual easy-difficult passage difference emerged as well.

From these analyses, it seems clear that the smaller familiarization effects that were significant in Experiment $2 \mathrm{~A}$ must be accepted with caution. If one uses a nonspecific familiarization control group, which itself did not yield a significant transfer effect, we see no reliable differences between treatment and control conditions. It may simply be that the measure here is insensitive to such small amounts of specific transfer, but certainly these effects of past experience do not appear to be reliably specific to the particular experience given. The original effect, captured again in the replication group, is, however, reliably different from the control of unrelated experience. The nature of this effect is clarified by the "scrambled" comparisons.

Scrambled group. Similar comparisons of the scrambled group with each of the replication, script, auditory, and paraphrase groups yielded exactly the opposite outcome to that reported above. That is, the conditions main effects were not significant in any comparison, but the familiarization main effects and, critically, the Conditions by Familiarization interactions were significant in all comparisons, except with the replication group $[F(1,46)=8.50,8.33$, and 13.40 for script, auditory, and paraphrase groups, respectively; $p>.01$ in all cases]. For the replication-scrambled comparison, the effect of familiarization was significant $[F(1,46)=98.42$, $\mathrm{p}<.001]$, but this effect did not differ for the two conditions $(F<1)$. Perusal of the means (see Table 3) indicates clearly that the familiarization effect for the scrambled condition was as large in magnitude as that for the replication group. These effects were both larger than for any of the other comparison groups. In sum. mary, then, repetition of the same words in a different visual typography or different modality did not lead to a replication of the original familiarization effect. Similarly, repetition of meaning, but not wording, did not capture the original effect. However, repeating the same words in the same script, even though all of the surrounding context words had been scrambled, yielded a familiarization or transfer effect equivalent to that of the original demonstration conditions.

These results were unexpected, as were two other findings: Namely, the scrambled condition, in which subjects were proofreading randomly arranged words, also yielded both better detection of errors in scram. bled easy (mean $=.71$ ) than in scrambled difficult (mean $=.64$ ) passages, and errors were again more poorly detected in function words (mean $=.64$ ) than in content words. despite the syntactic scrambling (means $=.70, .68$, and .69 for nouns, verbs, and adjectives, respectively). These two effects, ease of reading and comprehension and syntactic word class, had been used in Experiment 1 to suggest that subjects were indeed "reading" the proofreading pages at the syntactic and semantic levels of analysis. The occurrence of these effects in scrambled text, however, indicates that they are not being mediated by higher order constraints, but rather may represent the ease of visual analysis of words differing in certain properties. The passages used in the present studies were chosen to differ globally in ease of reading, and therefore differed in vocabulary level, as well as in syntactic and semantic structure. No attempt was made to control word properties of the error words across syntactic class or passage type. A post hoc analysis of the error words for the four easy and difficult passages of Set 1 , and the four children's tales, gives an indication of the word level differences. As Table 5 indicates, the words from the easy passages and the children's tales had fewer letters and fewer syllables than the words of the difficult passages. Further, since the easy passages and tales were both taken from children's books, whereas the difficult passages were from senior level university textbooks, the words necessarily differed in familiarity, frequency, and concrete-

Table 5

\begin{tabular}{|c|c|c|c|c|c|c|}
\hline \multirow{2}{*}{$\begin{array}{c}\text { Syntactic } \\
\text { Class }\end{array}$} & \multicolumn{2}{|c|}{ Tales } & \multicolumn{2}{|c|}{ Easy } & \multicolumn{2}{|c|}{ Difficult } \\
\hline & Median & Range & Median & Range & Median & Range \\
\hline \multicolumn{7}{|c|}{ (A) Letter Length of Error Words } \\
\hline Function & 4 & $2-9$ & 4 & $2-7$ & 3 & $2-8$ \\
\hline Noun & 7.5 & $5-11$ & 7 & $4-12$ & 10.5 & $5-17$ \\
\hline Adjective & 6 & $3-11$ & 6 & $3-9$ & 10 & $4-14$ \\
\hline Verb & 7 & $3-11$ & 7 & $5-10$ & 8 & $3-12$ \\
\hline \multicolumn{7}{|c|}{ (B) Number of Syllables of Error Words } \\
\hline Function & 1 & $1-3$ & 1 & $1 \cdot 2$ & 1 & $1-2$ \\
\hline Noun & 2 & $1-4$ & 2 & $1-3$ & 4 & $1-5$ \\
\hline Adjective & 2 & 14 & 2 & $1-3$ & 3 & $1-6$ \\
\hline Verb & 2 & $1-3$ & 2 & $1-4$ & 2 & $1-5$ \\
\hline
\end{tabular}


ness (e.g., pasture or princess vs. components or expectancy). Thus, the effects observed for variations in passage type were probably attributable to properties at the word, rather than at the syntactic or semantic, levels.

Similarly, as Haber and Schindler (1981) have already pointed out, comparisons across syntactic classes are filled with the natural language confoundings of frequency, familiarity, and length with the syntactic class of a word. Thus, comparisons across types of materials as they occur naturally, as opposed to artificially constrained texts, may lead to what appear to be "higher level" constraints on visual processing. These effects may, however, have more to do with the rapid visual analyses of familiar, shorter words, than with any syntactic or semantic constraints on on-line visual analyses.

\section{GENERAL DISCUSSION}

The experiments described here were designed to examine the transfer of specific prior knowledge from one reading of a text to another reading of the same text. Specifically, interest was in those levels of analyses that might constrain or alter the visual processes conducted during the second reading task. Two findings were of particular interest: (1) Contrary to the intuition that it is harder to proofread a familiar text, we report evidence that familiarity leads to a better revelation of spelling errors, even though these errors do not change the visual shape of the word. (2) This facilitation in subsequent proofreading seems highly specific to the reinstatement of the exact text that was previously read. Any change from the prior experience (typescript, modality changes, or wording changes that maintained meaning) led to a decrease in the advantage of the familiarization experience. This advantage was clearly not being mediated at the syntactic or semantic level, since typescript and modality changes that maintained the syntactic-semantic information lost the advantage, whereas scrambling the context words that destroyed the syntactic-semantic constraints maintained the familiarization advantage. Similarly, preserving meaning, but not wording, did not lead to a reliable specific facilitation in proofreading. Rather, only the re-presertation of the exact visual words, either in or out of context, led to a reliable facilitation in the reprocessing of those words, as measured by ability to see distortions in the word spellings. The phenomenon described by these experiments clearly does not succumb to a "top-down" processing view, in which higher level syntactic-semantic processes guided analyses at the letter and word levels. Rather, very specific processes at the letter and word levels are implicated.

Attributing the familiarization effect to lower, rather than higher, levels of processing does not, of course, specify the mechanisms involved in the facilitation. Does this familiarization effect tell us anything about the visual processing involved in reading? It is possible that the effects found here are specific to proofreading and do not reflect analyses normally used in reading. That is, the task's emphasis on speeded error detection, rather than on comprehension, may have created a "set" that "caused" the lower level processing observed here. Subjects may have "scanned," rather than "read," the familiar passages. Tasks placing more emphasis on comprehension may be less susceptible to these specific visual transfer effects. Such changes in processing, with changes in task demands, have been observed in listening comprehension (Cole \& Jakimik, 1980; Marslen-Wilson \& Welsh, 1978) and in the eye movement work (Ehrlich \& Rayner, 1981; Zola, Note 1). It is precisely these changes in the use of processes with different task demands that should offer insight into the interactive use of parallel processing systems. Reading is a complex skill that takes many forms, from scanning newspapers for current issues to thorough analysis of technical documents. As emphasized previously (Levy, 1981), a complete reading theory must incorporate the task demands and resource limitations that seem to give rise to a very flexible use of the processing system.

The familiarity effects observed in the present study suggest a surprising level of visual specificity in text processing. If one characterizes this specificity of transfer from prior reading to proofreading in terms of a static visual icon, then this iconic representation includes an enormous amount of information that remains available over many minutes and across many eye movements. These are not characteristics normally associated with iconic representation. If, on the other hand, the effect is thought of as visual scanning, then the scanning mode (familiarized proofreading) actually yields more complete visual information than does reading (unfamiliarized proofreading), since proofreading improves with familiarity. Again, this finding seems inconsistent with a view of scanning in terms of speedaccuracy tradeoff. That is, that the subject skims the page quickly, but at some cost to the thoroughness of the analyses of the material.

Finally, the familiarization effect does not easily succumb to a view suggesting that the reading experience somehow "primes" or "reminds" the reader of the error words' visual representation. More specifically, since students are often poor spellers, reading an errorfree text may "remind" the subjects of a word's spelling, so that during subsequent proofreading he will note the distortion. Three pieces of evidence argue against this view: (1) The errors all led to the creation of nonwords (e.g., rbymes or smoofly). The distortions were not subtle and it is unlikely that any university student would have classified the error words as English words. Thus, the detection task could be accomplished on a word-nonword discrimination, without need to "know" the correct spelling. (2) In the script condition, the subjects were equally familiarized with the words' spelling, 
yet the specific familiarization effect was not replicated. (3) The words in the children's tales and easy passages were at a child's reading level and should present no spelling problems to university students. The difficult passages contained words that were more likely to benefit from spelling practice. Yet, there was no interaction of passage type with the familiarization effect, suggesting that spelling skill was not critical. Similarly, the failure to find an interaction of familiarity with syntactic class, when function words present no spelling problems, argues against a spelling skills view. Taken together, these findings do not seem consistent with a spelling practice explanation of the familiarization effect.

Two possible explanations of the effect will be explored. First, Kolers et al. (1980) have suggested that prior experience with specific visual letter-sequence or word patterns will lead to positive transfer when those patterns are encountered in later text processing. They argued that this transfer occurs at the level of visual operations, since the patterning effect disappeared when changes were made in the visual display (e.g., when letters were separated by spaces or when the typescript was changed). The familiarization effect observed here also appears to be specific to the visual display experienced, since a change in typescript from reading to proofreading led to a loss of the familiarization effect. By this explanation, then, very specific visual "memories" or "operations" must be postulated for each word processed in the text. The word or even letter level is implicated by the fact that the familiarization effect was equally large for words that were again presented in the original text or in a scrambled arrangement. This view, then, would suggest that prior experience in some way "primes" visual processing skills or makes available specific visual representations to allow more fluent reanalyses and, thus, allows better detection of deviations from "known" patterns.

An alternative account would suggest that familiarity or prior knowledge does not change the fluency of any process, but rather leads to a redistribution of processing resources. Under normal reading conditions, processing resources may be distributed among all the levels of analyses required for reading, from word recognition to text integration and comprehension. When readers are familiar with a passage, fewer resources will be required to comprehend the "known" message, so that resources are "freed" for more analyses at the visual level. These analyses will then allow better detection of errors at the letter and word levels. A similar "freeing" of resources from higher level analyses will occur when a text is scrambled, since then no syntactic or semantic processing is possible. Thus, resources are available for visual word-level analyses when reading "scrambled" text, just as when reading very familiar text. By this view, then, prior knowledge leads to a better reading because more resources become available for lower level analyses, rather than through any priming of past memories or operations. Processing specificity is required, however, since this redistribution of resources appears to be disrupted by any change in the textual display. Typescript and modality changes lead to a loss in the familiarity effect, even though these texts are familiar to higher level processes. Thus, some interaction of data and conceptual knowledge determines the distribution of available resources. The interaction can be conceived of in terms of ease of higher level processing, rather than of higher processes constraining or guiding lower processes.

The present data cannot choose between the specific visual skills and the processing resource accounts, and indeed these may not be mutually exclusive alternatives. One potential argument against the processing resource account is that in the unfamiliarized scrambled case, there is no need for syntactic-semantic processing, unlike all other unfamiliarized conditions, so that subjects should have more processing resources available to proofread here. Yet, this condition yields the lowest level of proofreading. It should be noted, however, that even individual words require semantic processing. One must "read" the unfamiliar scrambled words. The familiarization experience facilitates higher level processing at the word level as well, thus freeing up resources to fully analyze the visual display. The general point, then, is that prior knowledge may constrain or alter subsequent visual analyses, not because higher level schema "guide" later visual processes, but rather because prior knowledge "frees up" processes resources to allow fuller visual analyses. This "attentional" or "processing resources" account differs from that of Haber and Schindler (1981), in that they suggest that prior knowledge leads to fewer visual analyses and, therefore, poorer error detection. In our task, the prior knowledge appears to provide more visual processing and, therefore, better error detection. Similarly, the facilitation observed here does not succumb to Healy's (1980) automatic processing account, since familiarity did affect proofreading, contrary to Healy's prediction. In summary, then, the data reported here reveal an aspect of prior knowledge constraint on subsequent visual processing, different from those previously reported. The theoretical account of reading suggested would require flexibility in the distribution of processing resources, given task demands, and familiarity at both the higher syntactic-semantic levels and the visually specific levels of analysis.

\section{REFTRENCE NOTE}

1. Zola, D. The effect of redundancy on the perception of words in reading. Manuscript submitted for publication, 1981.

\section{REFERENCES}

Cole, R. A., \& JAkimiK, J. Understanding speech: How words 
are heard. In G. Underwood (Ed.), Strategies of information processing. New York: Academic Press, 1978.

Cole, R. A., \& Jakimik, J. A model of speech perception. In R. A. Cole (Ed.), Perception and production of fluent speech. Hillsdale, N.J: Erlbaum, 1980.

Cole, R. A., \& Perfetti, C. A. Listening for mispronunciations in a children's story: The use of context by children and adults. Journal of Verbal Learning and Verbal Behavior, 1980, 19, 297-315.

Drewnowski, A., \& Healy, A. F. Detection of errors on the and: Evidence for reading units larger than the word. Memory \& Cognition, 1977, 5, 636-647.

Ehrlich, S. F., \& RAYNer, K. Contextual effects on word perception and eye movements during reading. Journal of Verbal Learning and Verbal Behavior, 1981, 20, 641-655.

HABER, R. N., \& Schindle R, R. M. Errors in proofreading: Evidence of syntactic control of letter processing? Journal of Experimental Psychology: Human Perception and Performance, 1981, 7, 573-579.

HeAly, A. F. Detection errors on the word the: Evidence for reading units longer than letters. Journal of Experimental Psychology: Human Perception and Performance, 1976, 2 , 235-242.

Healy, A. F. Proofreading errors on the word the: New evidence on reading units. Journal of Experimental Psychology: Human Perception and Performance, 1980, 6, 45-57.

Kolens, P. A., \& MAGEe, L. E. Specificity of pattern-analyzing skills in reading. Canadian Journal of Psychology, 1978, 32, 43-51.

Kolers, P. A., Palef, S. R., \& Stelmach, L. B. Graphemic analysis underlying literacy. Memory \& Cognition, 1980, 8, 322-328.

Krevger, L. E., Familiarity effects in visual information processing. Psychological Bulletin, 1975, 82, 949-974.
Kreuger, L. E., \& Weiss, M. E. Letter search through words and nonwords: The effect of fixed, absent, or mutilated targets. Memory \& Cognition, 1976, 4, 200-206.

KReUger, L. E., \& Shapiro, R. G. Letter detection with rapid serial visual presentation: Evidence against word superiority at feature extraction. Journal of Experimental Psychology: Human Perception and Performance, 1979, 5, 657-673.

LEVY, B. A. Interactive processing during reading. In A. M. Lesgold \& C. A. Perfetti (Eds.), Interactive processes in reading. Hillsdale, N.J: Erlbaum, 1981.

Marslen-Wilson, W. D., \& Welsh, A. Processing interactions at lexical access during word recognition in continuous speech. Cognitive Psychology, 1978, 10, 29-63.

McConkie, G. W., \& ZolA, D. Language constraints and the functional stimulus in reading. In A. M. Lesgold \& C. A. Perfetti (Eds.), Interactive processes in reading. Hillsdale, N.J: Erlbaum, 1981.

REicher, G. M. Perceptual recognition as a function of meaningfulness of stimulus material. Journal of Experimental Psychology, 1969, 81, 274-280.

RUMELhART, D. E. Toward an interactive model of reading. In S. Dornic (Ed.), Attention and performance VI. Hillsdale, N.J: Erlbaum, 1977.

Rumelhart, D. E., \& MCClelland, J. L. An interactive activation model of context effects in letter perception: Part 2. The contextual enhancement effect and some tests and extensions of the model. Psychological Review, 1982, 89, 60-94.

Schindler, R. M. The effect of prose context on visual search for letters. Memory \& Cognition, 1978, 6, 124-130.

Whemler, D. Processes in word recognition. Cognitive Psychology, 1970, 1, 59-85.

(Received for publication February 16, 1982; revision accepted July 28,1982 .) 